\title{
A HARDY SPACE ANALYSIS OF THE BÁEZ-DUARTE CRITERION FOR THE RH
}

\author{
S. WALEED NOOR
}

\begin{abstract}
In this article, methods from sub-Hardy Hilbert spaces such as the de Branges-Rovnyak spaces and local Dirichlet spaces are used to investigate Báez-Duarte's Hilbert space reformulation of the Riemann hypothesis (RH).
\end{abstract}

\section{INTRODUCTION}

A classical reformulation of the Riemann hypothesis by Nyman and Beurling (see [3], 16]) says that all the non-trivial zeros of the $\zeta$-function lie on the critical line $\operatorname{Re}(s)=1 / 2$ if and only if the characteristic function $\chi_{(0,1)}$ belongs to the closed linear span in $L^{2}((0,1))$ of the set $\left\{f_{\lambda}: 0 \leq \lambda \leq 1\right\}$, where $f_{\lambda}(x)=\{\lambda / x\}-\lambda\{1 / x\}$ (here $\{x\}$ is the fractional part). Almost fifty years later a remarkable strengthening of this result by Báez-Duarte [5] shows that we may replace $\lambda \in(0,1)$ by $\lambda=1 / \ell$ for $\ell \geq 2$. There is an equivalent version of the Báez-Duarte criterion in the weighted sequence space $\ell_{\omega}^{2}$ with inner product given by

$$
\langle x, y\rangle=\sum_{n=1}^{\infty} \frac{x(n) \overline{y(n)}}{n(n+1)}
$$

for sequences $x, y \in \mathcal{H}$ (see [7, page 73]). For each $k \geq 2$, let $r_{k}$ denote the sequence defined by $r_{k}(n)=k\{n / k\}$. Then the Báez-Duarte criterion may be stated as follows:

Theorem 1. The $R H$ is true if and only if $\mathbf{1}:=(1,1,1, \ldots)$ belongs to the closure of the linear span of $\left\{r_{k}: k \geq 2\right\}$ in $\ell_{\omega}^{2}$.

The plan of the paper is the following. Let $\mathcal{N}$ denote the linear span of the functions

$$
h_{k}(z)=\frac{1}{1-z} \log \left(\frac{1+z+\ldots+z^{k-1}}{k}\right)
$$

for $k \geq 2$, which all belong to the Hardy space $H^{2}$ (see Lemma 7 ). In Section 2 a unitary equivalent version of Theorem 1 for the Hardy space $H^{2}$ is presented. In particular, the $\mathrm{RH}$ holds if and only if the constant 1 belongs to the closure of $\mathcal{N}$ in $H^{2}$ (see Theorem 6). Section 3 introduces a multiplicative semigroup of weighted composition operators $\left\{W_{n}: n \geq 1\right\}$ on $H^{2}$ and shows that the constant 1 (appearing in Theorem 6) may be replaced by any cyclic vector for $\left\{W_{n}: n \geq 1\right\}$ in $H^{2}$. It follows that the $\mathrm{RH}$ is equivalent to the density of $\mathcal{N}$ in $H^{2}$ (see Theorem 8). Section 4 proves that $(I-S) \mathcal{N}$ is dense in $H^{2}$, where $S$ is the shift operator on $H^{2}$ (see Theorem 9). This central result has the following remarkable consequence.

Key words and phrases. Riemann hypothesis, Hardy space, Dirichlet space, de BrangesRovnyak space, Dilation completeness problem. 
That $\mathcal{N}$ is dense in $H^{2}$ with respect to the compact-open topology (see Theorem 10). Since convergence in $H^{2}$ implies convergence in the compact-open topology, this may be viewed as a weak form of the RH. Section 5 shows that $\mathcal{N}^{\perp}$ is in a sense small by proving that

$$
\mathcal{N}^{\perp} \cap \mathcal{D}_{\delta_{1}}=\{0\}
$$

where $\mathcal{D}_{\delta_{1}}$ is the local Dirichlet space at 1 (which is dense in $H^{2}$ ), and in particular that $\mathcal{N}^{\perp}$ contains no function holomorphic on a neighborhood of the closed unit disk $\overline{\mathbb{D}}$ (see Theorem 12). Section 6 shows that the cyclic vectors for $\left\{W_{n}: n \geq 1\right\}$ in $H^{2}$ are properly embedded into the set of all 2-periodic functions $\phi$ on $(0, \infty)$ having the property that the span of its dilates $\{\phi(n x): n \geq 1\}$ is dense in $L^{2}(0,1)$ (see Theorem [13). The characterization of all such $\phi$ is a famous open problem known as the Periodic Dilation Completeness Problem.

\section{BACKGROUND}

1.1. The Hardy-Hilbert space. We denote by $\mathbb{D}$ and $\mathbb{T}$ the open unit disk and the unit circle respectively. A holomorphic function $f$ on $\mathbb{D}$ belongs to the HardyHilbert space $H^{2}$ if

$$
\|f\|_{H^{2}}=\sup _{0 \leq r<1}\left(\frac{1}{2 \pi} \int_{0}^{2 \pi}\left|f\left(r e^{i \theta}\right)\right|^{2} d \theta\right)^{1 / 2}<\infty .
$$

The space $H^{2}$ is a Hilbert space with inner product

$$
\langle f, g\rangle=\sum_{n=0}^{\infty} a_{n} \overline{b_{n}}
$$

where $\left(a_{n}\right)_{n \in \mathbb{N}}$ and $\left(b_{n}\right)_{n \in \mathbb{N}}$ are the Maclaurin coefficients for $f$ and $g$ respectively. Similarly $H^{\infty}$ denotes the space of bounded holomorphic functions defined on $\mathbb{D}$. For any $f \in H^{2}$ and $\zeta \in \mathbb{T}$, the radial limit $f^{*}(\zeta):=\lim _{r \rightarrow 1^{-}} f(r \zeta)$ exists $m$-a.e. on $\mathbb{T}$, where $m$ denotes the normalized Lebesgue measure on $\mathbb{T}$.

1.2. A weighted Bergman space. Let $\mathcal{A}$ be the Hilbert space of analytic functions $f(z)=\sum_{n=0}^{\infty} a_{n} z^{n}$ and $g(z)=\sum_{n=0}^{\infty} b_{n} z^{n}$ defined on $\mathbb{D}$ for which the inner product is given by

$$
\langle f, g\rangle:=\sum_{n=0}^{\infty} \frac{a_{n} \overline{b_{n}}}{(n+1)(n+2)} .
$$

There also exists an area integral form of the corresponding $\mathcal{A}$-norm given by

$$
\|f\|_{\mathcal{A}}^{2}=\int_{\mathbb{D}}|f(z)|^{2}\left(1-|z|^{2}\right) d A(z)
$$

where $d A$ is the normalized area measure on $\mathbb{D}$. Comparing (0.1) with (1.1) shows that the map

$$
\Psi:(x(1), x(2), \ldots) \longmapsto \sum_{n=0}^{\infty} x(n+1) z^{n}
$$

is a canonical isometric isomorphism of $\ell_{\omega}^{2}$ onto $\mathcal{A}$. 
The text [12] is a modern reference for such weighted Bergman spaces. Also if $\operatorname{Hol}(\mathbb{D})$ is the space of all holomorphic functions on $\mathbb{D}$ and $T: \operatorname{Hol}(\mathbb{D}) \rightarrow \operatorname{Hol}(\mathbb{D})$ is an operator defined by

$$
\operatorname{Tg}(z):=\frac{((1-z) g(z))^{\prime}}{1-z}
$$

then $T$ restricted to $H^{2}$ is an isometric isomorphism onto $\mathcal{A}$ (see Lemma 7.2.3 [13]). Hence $\Phi:=T^{-1} \circ \Psi$ is an isometric isomorphism of $\ell_{\omega}^{2}$ onto $H^{2}$. Therefore to obtain a reformulation of the Báez-Duarte Theorem in $H^{2}$, we need to calculate $\Phi \mathbf{1}$ and $\Phi r_{k}$ for $k \geq 2$. But to do so we shall need some results about local Dirichlet spaces.

1.3. Generalized Dirichlet spaces. Let $\mu$ be a finite positive Borel measure on $\mathbb{T}$, and let $P \mu$ denote its Poisson integral. The generalized Dirichlet space $\mathcal{D}_{\mu}$ consists of $f \in H^{2}$ satisfying

$$
\mathcal{D}_{\mu}(f):=\int_{\mathbb{D}}\left|f^{\prime}(z)\right|^{2} P \mu(z) d A(z)<\infty .
$$

Then $\mathcal{D}_{\mu}$ is a Hilbert space with norm $\|f\|_{\mathcal{D}_{\mu}}^{2}:=\|f\|_{2}^{2}+\mathcal{D}_{\mu}(f)$. If $\mu=m$, then $\mathcal{D}_{m}$ is the classicial Dirichlet space. If $\mu=\delta_{\zeta}$ is the Dirac measure at $\zeta \in \mathbb{T}$, then $\mathcal{D}_{\delta_{\zeta}}$ is called the local Dirichlet space at $\zeta$ and in particular

$$
\mathcal{D}_{\delta_{\zeta}}(f)=\int_{\mathbb{D}}\left|f^{\prime}(z)\right|^{2} \frac{1-\left|z^{2}\right|}{|z-\zeta|^{2}} d A(z) .
$$

The recent book [13] contains a comprehensive treatment of local Dirichlet spaces and the following result establishes a criterion for their membership.

Theorem 2. (See [13, Thm. 7.2.1]) Let $\zeta \in \mathbb{T}$ and $f \in \operatorname{Hol}(\mathbb{D})$. Then $\mathcal{D}_{\delta_{\zeta}}(f)<\infty$ if and only if

$$
f(z)=a+(z-\zeta) g(z)
$$

for some $g \in H^{2}$ and $a \in \mathbb{C}$. In this case $\mathcal{D}_{\delta_{\zeta}}(f)=\|g\|_{2}^{2}$ and

$$
a=f^{*}(\zeta):=\lim _{r \rightarrow 1^{-}} f(r \zeta)
$$

Each local Dirichlet space $\mathcal{D}_{\delta_{\zeta}}$ is a proper subspace of $H^{2}$ and it has the distinctive property that evaluation at the boundary $f \mapsto f^{*}(\zeta)$ is a bounded linear functional [13, Thm. 8.1.2 (ii)].

1.4. The de Branges-Rovnyak spaces. Given $\psi \in L^{\infty}(\mathbb{T})$, the corresponding Toeplitz operator $T_{\psi}: H^{2} \rightarrow H^{2}$ is defined by

$$
T_{\psi} f:=P_{+}(\psi f)
$$

where $P_{+}: L^{2}(\mathbb{T}) \rightarrow H^{2}$ denotes the orthogonal projection of $L^{2}(\mathbb{T})$ onto $H^{2}$. Clearly $T_{\psi}$ is a bounded operator on $H^{2}$ with $\left\|T_{\psi}\right\| \leq\|\psi\|_{L^{\infty}}$. If $h \in H^{\infty}$, then $T_{h}$ is simply the operator of multiplication by $h$ and its adjoint is $T_{\bar{h}}$. Given $b$ in the closed unit ball of $H^{\infty}$, the de Branges-Rovnyak space $\mathcal{H}(b)$ is the image of $H^{2}$ under the operator $\left(I-T_{b} T_{\bar{b}}\right)^{1 / 2}$. A norm is defined on $\mathcal{H}(b)$ making $\left(I-T_{b} T_{\bar{b}}\right)^{1 / 2}$ a partial isometry from $H^{2}$ onto $\mathcal{H}(b)$. If $b \equiv 0$ then $\mathcal{H}(b)=H^{2}$, and if $b$ is inner then $\mathcal{H}(b)=\left(b H^{2}\right)^{\perp}$ is the model subspace of $H^{2}$. The recent two-volume work (9] 10]) is an encyclopedic reference for these spaces.

The general theory of $\mathcal{H}(b)$ spaces divides into two distinct cases, according to whether $\mathrm{b}$ is an extreme point or a non-extreme point of the unit ball of $H^{\infty}$. 
We shall only be concerned with the non-extreme case which is best illustrated by the next result (see [9, Chapter 6] and [18, Sects. IV-6 and V-1]).

Theorem 3. Let $b \in H^{\infty}$ with $\|b\|_{H^{\infty}} \leq 1$. The following are equivalent:

(1) $b$ is a non-extreme point of the unit ball of $H^{\infty}$,

(2) $\log \left(1-\left|b^{*}\right|^{2}\right) \in L^{1}(\mathbb{T})$,

(3) $\mathcal{H}(b)$ contains all functions holomorphic in a neighborhood of $\overline{\mathbb{D}}$.

When $b$ is non-extreme there exists a unique outer function $a \in H^{\infty}$ such that $a(0)>0$ and $\left|a^{*}\right|^{2}+\left|b^{*}\right|^{2}=1$ a.e. on $\mathbb{T}$. In this situation $(b, a)$ is usually called a pair and the function $b / a$ belongs to the Smirnov class $N^{+}$of quotients $p / q$ where $p, q \in H^{\infty}$ and $q$ is an outer function. That all $N^{+}$functions arise as the quotient of a pair associated to a non-extreme function was shown by Sarason (see [17]).

In 17, Sarason also demonstrated how $\mathcal{H}(b)$ spaces appear naturally as the domains of some unbounded Toeplitz operators. Let $\varphi$ be holomorphic in $\mathbb{D}$ and $T_{\varphi}$ the operator of multiplication by $\varphi$ on the domain

$$
\operatorname{dom}\left(T_{\varphi}\right)=\left\{f \in H^{2}: \varphi f \in H^{2}\right\} .
$$

Then $T_{\varphi}$ is a closed operator, and $\operatorname{dom}\left(T_{\varphi}\right)$ is dense in $H^{2}$ if and only if $\varphi \in N^{+}$ (see [17, Lemma 5.2]). In this case its adjoint $T_{\varphi}^{*}$ is also densely defined and closed. In fact the domain of $T_{\varphi}^{*}$ is a de Branges-Rovnyak space.

Theorem 4. (See [17, Prop. 5.4]) Let $\varphi$ be a nonzero function in $N^{+}$with $\varphi=b / a$, where $(b, a)$ is the associated pair. Then $\operatorname{dom}\left(T_{\varphi}^{*}\right)=\mathcal{H}(b)$.

If $\varphi$ is a rational function in $N^{+}$the corresponding pair $(b, a)$ is also rational (see [17. Remark. 3.2]). Recently Constara and Ransford [8] characterized the rational pairs $(b, a)$ for which $\mathcal{H}(b)$ is a generalized Dirichlet space.

Theorem 5. (See 8, Theorem 4.1]) Let $(b, a)$ be a rational pair and $\mu$ a finite positive measure on $\mathbb{T}$. Then $\mathcal{H}(b)=\mathcal{D}_{\mu}$ if and only if

(1) the zeros of a on $\mathbb{T}$ are all simple, and

(2) the support of $\mu$ is exactly equal to this set of zeros.

These ideas will be used in Section 5 to investigate the orthogonal complement of the functions $\left\{h_{k}: k \geq 2\right\}$ in $H^{2}$.

\section{The BÁEZ-DuARte CRITERION IN $H^{2}$}

The first main objective is to obtain a unitary equivalent version of Báez-Duarte's theorem (Theorem 11) in $H^{2}$ upon which to base the rest of our analysis.

Theorem 6. For each $k \geq 2$, define

$$
h_{k}(z)=\frac{1}{1-z} \log \left(\frac{1+z+\ldots+z^{k-1}}{k}\right) .
$$

Then the Riemann hypothesis holds if and only if the constant 1 belongs to the closed linear span of $\left\{h_{k}: k \geq 2\right\}$ in $H^{2}$.

In order to prove this, we must show that $-1=\Phi \mathbf{1}$ and $h_{k}=\Phi r_{k}$ for $k \geq 2$, where $\Phi:=T^{-1} \circ \Psi: \ell_{\omega}^{2} \rightarrow H^{2}$ is an isometric isomorphism (see subsection 1.2). 
We first find $R:=\Psi \mathbf{1}$ and $R_{k}:=\Psi r_{k}$, which belong to the weighted Bergman space $\mathcal{A}$. Then

$$
R(z)=\frac{1}{1-z}, \quad R_{k}(z)=\frac{1}{1-z}\left[\log \left(1+z+\ldots+z^{k-1}\right)\right]^{\prime}
$$

for each $k=2,3, \ldots$ (note that $R_{1} \equiv 0$ ). The expression for $R$ is trivial. For $R_{k}$ we first note that the sequence $r_{k}(n)=k\{n / k\}$ is periodic with $k$ distinct integer terms $\{1,2, \ldots, k-1,0, \ldots\}$. Hence collecting terms with common coefficients gives

$$
\begin{aligned}
R_{k}(z) & =\sum_{n=0}^{\infty} z^{n k}+2 \sum_{n=0}^{\infty} z^{n k+1}+\ldots+(k-1) \sum_{n=0}^{\infty} z^{n k+k-2}=\sum_{m=1}^{k-1} m \sum_{n=0}^{\infty} z^{n k+m-1} \\
& =\sum_{m=1}^{k-1} m \frac{z^{m-1}}{1-z^{k}}=\frac{1}{1-z^{k}} \sum_{m=1}^{k-1} m z^{m-1}=\frac{1}{1-z}\left[\frac{\left(1+z+\ldots+z^{k-1}\right)^{\prime}}{1+z+\ldots+z^{k-1}}\right] \\
(2.1) & =\frac{1}{1-z}\left[\log \left(1+z+\ldots+z^{k-1}\right)\right]^{\prime} .
\end{aligned}
$$

Next we calculate $T^{-1} R$ and $T^{-1} R_{k}$ in $H^{2}$. It is easy to see that $T(-1)=R$ and hence $-1=\Phi \mathbf{1}$. But finding the $T^{-1} R_{k}$ is not as straightforward because $T$ is not injective on $\operatorname{Hol}(\mathbb{D})$.

Lemma 7. For each non-zero $c$ and integer $k \geq 2$, define the function

$$
h_{k, c}(z)=\frac{1}{1-z} \log \left(\frac{1+z+\ldots+z^{k-1}}{c}\right) .
$$

Then $T h_{k, c}=R_{k}$ for each $c$, but $h_{k, c} \in H^{2}$ if and only if $c=k$.

Proof. Let $s_{k}(z):=\log \left(1+z+\ldots+z^{k-1}\right)$ for $k \geq 2$. Since $R_{k}$ belongs to $\mathcal{A}$ for $k \geq 2$, by (1.2), (1.5) and (2.1) we have

$$
\begin{aligned}
\mathcal{D}_{\delta_{1}}\left(s_{k}\right) & =\int_{\mathbb{D}}\left|\left[\log \left(1+z+\ldots+z^{k-1}\right)\right]^{\prime}\right|^{2} \frac{1-|z|^{2}}{|z-1|^{2}} d A(z) \\
& =\int_{\mathbb{D}}\left|R_{k}(z)\right|^{2}\left(1-|z|^{2}\right) d A(z) \\
& =\left\|R_{k}\right\|_{\mathcal{A}}^{2}<\infty .
\end{aligned}
$$

Therefore $s_{k}$ belongs to the local Dirichlet space $\mathcal{D}_{\delta_{1}}$. By Theorem 2 there exists $f_{k} \in H^{2}$ such that $s_{k}(z)=s_{k}^{*}(1)+(z-1) f_{k}(z)=\log k+(z-1) f_{k}(z)$ and it follows immediately that

$$
f_{k}(z)=\frac{1}{z-1} \log \left(\frac{1+z+\ldots+z^{k-1}}{k}\right) .
$$

Hence $h_{k, k}=-f_{k} \in H^{2}$. Since clearly $T h_{k, c}=R_{k}$ for each non-zero $c$ and $T$ is injective on $H^{2}$, therefore $c=k$ is the only value for which $h_{k, c} \in H^{2}$.

Therefore with $h_{k}:=h_{k, k}$ for all $k \geq 2$ this concludes the proof of Theorem 6 , We end this section by giving an alternate proof of the fact that $h_{k} \in H^{2}$ for $k>2$ which also provides an explicit formula for the Maclaurin coefficients of the $h_{k}$ 1]

\footnotetext{
${ }^{1}$ The author wishes to thank the anonymous referee for this alternate proof.
} 
For $k, n \in \mathbb{N}$, define the function $[k \mid n]$ to be 1 if $k$ divides $n$ and 0 otherwise, and note that $\log \left(1-z^{k}\right)=-\sum_{j \geq 1} z^{j k} / j=-\sum_{n \geq 1} k[k \mid n] z^{n} / n$. Then for $k \geq 2$

$$
\begin{aligned}
h_{k}(z) & =\frac{1}{1-z}\left(\log \left(1-z^{k}\right)-\log (1-z)-\log k\right) \\
& =\frac{1}{1-z}\left(-\sum_{n \geq 1} k[k \mid n] \frac{z^{n}}{n}+\sum_{n \geq 1} \frac{z^{n}}{n}-\log k\right) \\
& =-(\log k) \sum_{n \geq 0} z^{n}+\frac{1}{1-z} \sum_{n \geq 1} \frac{z^{n}}{n}(1-k[k \mid n])=\sum_{n \geq 0} c_{n}(k) z^{n}
\end{aligned}
$$

where $c_{n}(k)=-\log k+\sum_{j=1}^{n} \frac{1}{j}(1-k[k \mid j])=H(n)-H(n / k)-\log k$ and the function $H(x):=\sum_{n \leq x} \frac{1}{n}$ for $x>0$ and $H(0)=0$. The Euler-Maclaurin summation formula gives

$$
H(x)=\log x+\lambda-\frac{\{x\}}{x}+\int_{x}^{\infty}\{t\} \frac{d t}{t^{2}}=\log x+\lambda+O\left(\frac{1}{x}\right)
$$

where $\lambda$ is Euler's constant and hence $c_{n}(k)=O\left(\frac{k}{n}\right)$. Therefore $h_{k} \in H^{2}$.

\section{A Weighted COMPosition SEMigroup}

In 2. Bagchi showed that in addition to Theorem 1] the $\mathrm{RH}$ is equivalent to the density of $\operatorname{span}\left\{r_{k}: k \geq 2\right\}$ in $\ell_{\omega}^{2}$. A key ingredient in his proof is a multiplicative semigroup of operators which leave $\operatorname{span}\left\{r_{k}: k \geq 2\right\}$ invariant (see [2, Theorem 7]). The relation of invariant subspaces of semigroups with the $\mathrm{RH}$ has been evident since the thesis of Nyman [16] (see also [6] and [15]).

For each $n \geq 1$, let $W_{n}$ be a weighted composition operator on $H^{2}$ defined by

$$
W_{n} f(z)=\left(1+z+\ldots+z^{n-1}\right) f\left(z^{n}\right)=\frac{1-z^{n}}{1-z} f\left(z^{n}\right) .
$$

Note that each $W_{n}$ is bounded on $H^{2}, W_{1}=I$ and $W_{m} W_{n}=W_{m n}$ for each $m, n \geq 1$. Hence $\left\{W_{n}: n \geq 1\right\}$ is a multiplicative semigroup on $H^{2}$. Now if we write

$$
h_{k}(z)=\frac{1}{1-z}\left(\log \left(1-z^{k}\right)-\log (1-z)-\log k\right)
$$

then it is easy to see that $W_{n} h_{k}=h_{n k}-h_{n}$ for all $k, n \geq 1$ (where $h_{1} \equiv 0$ ). Hence the linear span of $\left\{h_{k}: k \geq 2\right\}$ is invariant under $\left\{W_{n}: n \geq 1\right\}$. A vector $f \in H^{2}$ is called a cyclic vector for an operator semigroup $\left\{S_{n}: n \geq 1\right\}$ if $\operatorname{span}\left\{S_{n} f: n \geq 1\right\}$ is dense in $H^{2}$. Hence the following combines Bagchi's result and a generalization of Theorem 6 ,

Theorem 8. The following statements are equivalent

(1) The Riemann hypothesis,

(2) the closure of $\operatorname{span}\left\{h_{k}: k \geq 2\right\}$ contains a cyclic vector for $\left\{W_{n}: n \geq 1\right\}$,

(3) $\operatorname{span}\left\{h_{k}: k \geq 2\right\}$ is dense in $H^{2}$.

Proof. The equivalence (1) $\leftrightarrow(3)$ is just Bagchi's result transfered to $H^{2}$ via the isomorphism $\Phi: \ell_{\omega}^{2} \rightarrow H^{2}$. The implication (1) $\rightarrow$ (2) follows from Theorem [6 and the fact that 1 is a cyclic vector for the semigroup $\left\{W_{n}: n \geq 1\right\}$. Indeed $\left(W_{n} 1\right)(z)=1+z+\ldots+z^{n-1}$ for all $n \geq 1$ so $\operatorname{span}\left\{W_{n} 1: n \geq 1\right\}$ contains all analytic polynomials and is hence dense in $H^{2}$. Finally $(2) \rightarrow(3)$ because if the closure of $\operatorname{span}\left\{h_{k}: k \geq 2\right\}$ contains a cyclic vector $f \in H^{2}$, then it also contains 
the dense manifold $\operatorname{span}\left\{W_{n} f: n \geq 1\right\}$ by the invariance of $\operatorname{span}\left\{h_{k}: k \geq 2\right\}$ under $\left\{W_{n}: n \geq 1\right\}$.

In Section 6, we shall see that characterizing the cyclic vectors for $\left\{W_{n}: n \geq 1\right\}$ is intimately related to another famous open problem known as the Periodic Dilation Completeness Problem (see [11] and [15]).

\section{The Density of $\operatorname{span}\left\{(I-S) h_{k}: k \geq 2\right\}$ IN $H^{2}$}

Let $S=T_{z}$ be the shift operator on $H^{2}$. Since $I-S$ has dense range (because $I-S^{*}$ is injective), therefore $\operatorname{span}\left\{(I-S) h_{k}: k \geq 2\right\}$ is dense in $H^{2}$ under the $\mathrm{RH}$ by Theorem 8 . Proving that this statement is unconditionally true is the main objective of this section and it will play a central role in the rest of this work.

Theorem 9. The span of $\left\{(I-S) h_{k}: k \geq 2\right\}$ is dense in $H^{2}$.

Since convergence in $H^{2}$ implies uniform convergence on compact subsets of $\mathbb{D}$, we obtain a weak version of the $\mathrm{RH}$.

Theorem 10. The span of $\left\{h_{k}: k \geq 2\right\}$ is dense in $H^{2}$ with the compact-open topology.

Proof. The formal inverse of $I-S$ is the Toeplitz operator $T_{\varphi}$ of multiplication by the function $\varphi(z)=\frac{1}{1-z}$. Although $T_{\varphi}$ is unbounded on $H^{2}$ (otherwise Theorem 9 would imply the $\mathrm{RH}$ ), it is still continuous on $H^{2}$ with the compact-open topology. Therefore the result follows immediately from Theorem 9 .

Define the multiplicative operator semigroup $\left\{T_{n}: n \geq 1\right\}$ on $H^{2}$ by

$$
T_{n} f(z)=f\left(z^{n}\right) .
$$

Then by (3.1) and (4.1) it is easily seen that

$$
T_{n}(I-S)=(I-S) W_{n} \quad \forall n \geq 1 .
$$

Recall that $\operatorname{span}\left\{h_{k}: k \geq 2\right\}$ is invariant under $\left\{W_{n}: n \geq 1\right\}$ (see Section 3), and hence (4.2) implies that $\operatorname{span}\left\{(I-S) h_{k}: k \geq 2\right\}$ is invariant under $\left\{T_{n}: n \geq 1\right\}$. So to prove Theorem 9, it is enough to prove that the closure of $\operatorname{span}\left\{(I-S) h_{k}: k \geq 2\right\}$ contains a cyclic vector for $\left\{T_{n}: n \geq 1\right\}$. And the cyclic vector we consider is $1-z$. Indeed, if $f \in H^{2}$ is orthogonal to each $T_{n}(1-z)=1-z^{n}$ then $\widehat{f}(0)=\widehat{f}(n)$ for all $n \geq 1$ and hence $f \equiv 0$. Hence the next result completes the proof of Theorem 9 .

Lemma 11. The series $\sum_{k=2}^{\infty} \frac{\mu(k)}{k}(I-S) h_{k}$ converges to $1-z$ in $H^{2}$, where $\mu$ is the Möbius function.

Recall that the Möbius function is defined on $\mathbb{N}$ by $\mu(k)=(-1)^{s}$ if $k$ is the product of $s$ distinct primes, and $\mu(k)=0$ otherwise. In the proof we shall need the Prime Number Theorem in the equivalent forms

$$
\sum_{k=1}^{\infty} \frac{\mu(k)}{k}=0 \text { and } \sum_{k=1}^{\infty} \frac{\mu(k) \log k}{k}=-1
$$

(see [1, Thm. 4.16] and [14, p. 185, Excercise 16]). 
Proof. One has to prove that

$$
\left\|\sum_{k=2}^{n} \frac{\mu(k)}{k}(I-S) h_{k}+z-1\right\|_{H^{2}} \longrightarrow 0
$$

as $n \rightarrow \infty$. Since $(I-S) h_{k}(z)=\log \left(1-z^{k}\right)-\log (1-z)-\log k$ (see (3.2) $)$, we get

$$
\sum_{k=2}^{n} \frac{\mu(k)}{k}(I-S) h_{k}(z)=\sum_{k=1}^{n} \frac{\mu(k)}{k} \log \left(1-z^{k}\right)-\sum_{k=1}^{n} \frac{\mu(k)}{k}[\log (1-z)+\log k]
$$

First note that the last sum on the right of (4.5) tends to 1 as $n \rightarrow \infty$ by (4.3). Writing the first sum as a double sum after noting that $\log \left(1-z^{k}\right)=-\sum_{j=1}^{\infty} \frac{z^{j k}}{j}$, interchanging the order of summation and using the basic identity $\sum_{d \mid j} \mu(d)=\left[\frac{1}{j}\right]$ if $j \geq 1$ [1, Thm 2.1] ([x] denotes the integer part of $x$ ), we get

$$
\begin{aligned}
& \sum_{k=1}^{n} \frac{\mu(k)}{k} \log \left(1-z^{k}\right)=-\sum_{k=1}^{n} \frac{\mu(k)}{k} \sum_{j=1}^{\infty} \frac{z^{j k}}{j}=-\sum_{k=1}^{n} \mu(k) \sum_{j=1}^{\infty} \frac{z^{j k}}{j k} \\
& =-\sum_{j=1}^{\infty} \frac{z^{j}}{j} \sum_{\substack{d \mid j \\
1 \leq d \leq n}} \mu(d)=-\sum_{j=1}^{n} \frac{z^{j}}{j} \sum_{d \mid j} \mu(d)-\sum_{j=n+1}^{\infty} \frac{z^{j}}{j} \sum_{\substack{d \mid j \\
1 \leq d \leq n}} \mu(d) \\
& =-\sum_{j=1}^{n} \frac{z^{j}}{j}\left[\frac{1}{j}\right]-\sum_{j=n+1}^{\infty} \frac{z^{j}}{j} \sum_{\substack{d \mid j \\
1 \leq d \leq n}} \mu(d)=-z-\phi_{n}(z) .
\end{aligned}
$$

Therefore by (4.5) and (4.6), we will prove (4.4) once we prove that $\left\|\phi_{n}\right\|_{H^{2}} \rightarrow 0$ as $n \rightarrow \infty$. Since

$$
\phi_{n}(z)=\sum_{j=n+1}^{\infty} \frac{z^{j}}{j} \sum_{\substack{d \mid j \\ 1 \leq d \leq n}} \mu(d)
$$

and if $\sigma(n)$ denotes the number of divisors of $n$, then it follows that

$$
\left|\sum_{\substack{d \mid j \\ 1 \leq d \leq n}} \mu(d)\right| \leq \sum_{d \mid j} 1=\sigma(j) .
$$

The function $\sigma$ satisfies the relation $\sigma(n)=o\left(n^{\epsilon}\right)$ for every $\epsilon>0$ [1, p. 296]. In particular, $\sigma(n) \lesssim n^{\epsilon}$ for some $0<\epsilon<\frac{1}{2}$, and therefore by (4.7)

$$
\left\|\phi_{n}\right\|_{H^{2}}^{2} \leq \sum_{j=n+1}^{\infty} \frac{\sigma(j)^{2}}{j^{2}} \lesssim \sum_{j=n+1}^{\infty} j^{2 \epsilon-2} \longrightarrow 0
$$

as $n \rightarrow \infty$. This proves (4.4) and hence the lemma.

\section{Functions orthogonal to $\left\{h_{k}: k \geq 2\right\}$}

The RH is equivalent to $\left\{h_{k}: k \geq 2\right\}^{\perp}$ being trivial $\{0\}$ (see Theorem 8 ). The main result of this section shows that $\left\{h_{k}: k \geq 2\right\}^{\perp}$ is indeed in a sense very small. 
Theorem 12. We have

$$
\left\{h_{k}: k \geq 2\right\}^{\perp} \cap \mathcal{D}_{\delta_{1}}=\{0\}
$$

where $\mathcal{D}_{\delta_{1}}$ is the local Dirichlet space at 1 . In particular $\left\{h_{k}: k \geq 2\right\}^{\perp}$ contains no function holomorphic on a neighborhood of the closed unit disk $\overline{\mathbb{D}}$.

The key idea is to use the formal inverse $T_{\varphi}$ of $I-S$, where $\varphi(z)=\frac{1}{1-z}$ is clearly an $N^{+}$function. Then there is a pair $(b, a)$ associated with $\varphi$ where

$$
a(z)=\frac{\gamma(1-z)}{(\gamma+1)-z}
$$

and $\gamma=\frac{1+\sqrt{5}}{2}$ is the golden ratio (see [17, page 284]). Therefore by Theorem 4 Theorem 5 and (5.1) we immediately see that

$$
\operatorname{dom}\left(T_{\varphi}^{*}\right)=\mathcal{H}(b)=\mathcal{D}_{\delta_{1}}
$$

where $T_{\varphi}^{*}$ is the adjoint of $T_{\varphi}$ (see subsection 1.4).

Proof. Let $g_{k}:=(I-S) h_{k}$ for each $k \geq 2$ and note that $\operatorname{span}\left\{g_{k}: k \geq 2\right\}$ is dense in $H^{2}$ by Theorem 9 . Also note $g_{k} \in \operatorname{dom}\left(T_{\varphi}\right)$ because $h_{k}=T_{\varphi} g_{k}$ and by (1.6). Now let $p$ be an element in $\left\{h_{k}: k \geq 2\right\}^{\perp} \cap \operatorname{dom}\left(T_{\varphi}^{*}\right)$. Hence for each $k \geq 2$, we have

$$
\left\langle T_{\varphi}^{*} p, g_{k}\right\rangle=\left\langle p, T_{\varphi} g_{k}\right\rangle=\left\langle p, h_{k}\right\rangle=0 .
$$

Therefore $T_{\varphi}^{*} p \equiv 0$. But this implies that $p \equiv 0$, because

$$
\left\langle p, T_{\varphi} f\right\rangle=\left\langle T_{\varphi}^{*} p, f\right\rangle=0
$$

for each $f \in \operatorname{dom}\left(T_{\varphi}\right)$ and the range of $T_{\varphi}$ is all of $H^{2}$ (it is the domain of $I-S$ ). Hence $\left\{h_{k}: k \geq 2\right\}^{\perp} \cap \operatorname{dom}\left(T_{\varphi}^{*}\right)=\{0\}$, (5.2) and Theorem 3 complete the proof.

\section{The Periodic Dilation Completeness Problem PDCP}

The PDCP asks which 2-periodic functions $\phi$ on $(0, \infty)$ have the property that

$$
\operatorname{span}\{\phi(n x): n \geq 1\}
$$

is dense in $L^{2}(0,1)$. In this case we shall just say that $\phi$ is a PDCP function. This difficult open problem was first considered independently by Wintner [19] and Beurling [4. See [11] and [15] for beautiful modern treatments. The main result of this section shows that the cyclic vectors for $\left\{W_{n}: n \geq 1\right\}$ in $H^{2}$ (see Theorem 8) are properly embedded into the PDCP functions.

Theorem 13. There exists an injective linear map $V: H^{2} \rightarrow L^{2}(0,1)$ such that if $f$ is a cyclic vector for $\left\{W_{n}: n \geq 1\right\}$ in $H^{2}$, then $V f$ is a PDCP function.

The function $V f \in L^{2}(0,1)$ is defined on the whole real line by extending it as an odd 2-periodic function.

Proof. Recall that the semigroups $\left\{W_{n}: n \geq 1\right\}$ and $\left\{T_{n}: n \geq 1\right\}$ satisfy the relation

$$
T_{n}(I-S)=(I-S) W_{n} \quad \forall n \geq 1 .
$$

where $I-S$ has dense range in $H^{2}$ (see (4.2)). It follows that if $\operatorname{span}\left\{W_{n} f: n \geq 1\right\}$ is dense in $H^{2}$ for some $f \in H^{2}$, then $\operatorname{span}\left\{T_{n}(I-S) f: n \geq 1\right\}$ must also be dense. 
So $f \mapsto(I-S) f$ maps cyclic vectors for $\left\{W_{n}: n \geq 1\right\}$ to cyclic vectors for $\left\{T_{n}: n \geq 1\right\}$. Let

$$
H_{0}^{2}:=\left\{f \in H^{2}: f(0)=0\right\}=H^{2} \ominus \mathbb{C}
$$

and note that $H_{0}^{2}$ is a reducing subspace for $T_{n}$ since $T_{n} \mathbb{C} \subset \mathbb{C}$ and $T_{n} H_{0}^{2} \subset H_{0}^{2}$. Denote by $P$ the orthogonal projection of $H^{2}$ onto $H_{0}^{2}$. It follows that if $f$ is a cyclic vector for $\left\{T_{n}: n \geq 1\right\}$ in $H^{2}$ then $P f$ is a cyclic vector for $\left\{T_{n}: n \geq 1\right\}$ restricted to $H_{0}^{2}$. Therefore

$$
P(I-S): H^{2} \rightarrow H_{0}^{2}
$$

maps cyclic vectors for $\left\{W_{n}: n \geq 1\right\}$ into cyclic vectors for $\left\{T_{n}: n \geq 1\right\}$ restricted to $H_{0}^{2}$. Finally there is a unitary operator $U: H_{0}^{2} \rightarrow L^{2}(0,1)$ such that $f$ is cyclic for $\left\{T_{n}: n \geq 1\right\}$ in $H_{0}^{2}$ if and only if $U f$ is a PDCP function (see [15, page 1707]). In fact, it is defined by

$$
U: z^{k} \longmapsto e_{k}(x):=\sqrt{2} \sin (\pi k x)
$$

for each $k \geq 1$, where $\left(e_{k}\right)_{k \geq 1}$ is an orthonormal basis for $L^{2}(0,1)$. Therefore the operator

$$
V:=U P(I-S): H^{2} \rightarrow L^{2}(0,1)
$$

maps cyclic vectors for $\left\{W_{n}: n \geq 1\right\}$ into PDCP functions. It is injective since $\operatorname{Ker}(P)=\mathbb{C}$ and the inverse image of $\mathbb{C}$ under $I-S$ is $\{0\}$.

Finally, we show that not all PDCP functions belong to the range of $V$ (6.2). Wintner [19] showed that for $\operatorname{Re}(s)>1 / 2$ the function

$$
f_{s}(x)=\sum_{k \geq 1} k^{-s} \sqrt{2} \sin (\pi k x)
$$

is a PDCP function. We give an independent proof that $f_{1}$ is a PDCP function and that it does not belong to the range of $V$.

Theorem 14. $f_{1}$ is a PDCP function that does not belong to the range of $V$.

Proof. Let $L(z):=\log (1-z)=-\sum_{k \geq 1} z^{k} / k$ and note that $U(-L)=f_{1}$ (see (6.1)). Hence it is enough to prove that $L$ is a cyclic vector for $\left\{T_{n}: n \geq 1\right\}$ in $H_{0}^{2}$. Note that since $(I-S) h_{k}(z)=\log \left(1-z^{k}\right)-\log (1-z)-\log k$ we have

$$
P(I-S) h_{k}=T_{k} L-T_{1} L
$$

and hence

$$
P\left(\operatorname{span}\left\{(I-S) h_{k}: k \geq 2\right\}\right) \subset \operatorname{span}\left\{T_{n} L: n \geq 1\right\} .
$$

By Theorem 9 the left side of (6.3) is dense in $H_{0}^{2}$ and hence $L$ is cyclic. Therefore $f_{1}$ is a PDCP function. To prove that $f_{1}$ is not in the range of $V$, we show that $L$ is not in the range $P(I-S)$. The functions mapped onto $L$ by $P$ are of the form $\alpha+L$ for some $\alpha \in \mathbb{C}$. But $\alpha+L$ does not belong to $(I-S) H^{2}$ because $L^{*}(1)$ does not exist and $f^{*}(1)=0$ for all $f \in(I-S) H^{2}$ (see Theorem 2).

\section{ACKNOWLEDGEMENT}

This work has been partially supported by a FAPESP grant (17/09333-3). 


\section{REFERENCES}

1. T. M. Apostol, Introduction to analytic number theory. UTM Springer, 1976.

2. B. Bagchi, On Nyman, Beurling and Báez-Duarte's Hilbert space reformulation of the Riemann hypothesis. Proc. Ind. Acad. Sci (Math. Sci.), 116(2), 137-146, 2006.

3. A. Beurling, A closure problem related to the Riemann zeta-function. Proc. Nat. Acad. Sci., 41, 312-314, 1955

4. A. Beurling, On the completeness of $\psi(n t)$ on $L^{2}(0,1)$, in Harmonic Analysis, Contemp. Mathematicians, The collected works of Arne Beurling, vol. 2, Birkhauser, Boston, 1989, p. 378-380.

5. L. Báez-Duarte, A strengthening of the Nyman-Beurling criterion for the Riemann hypothesis. Atti Acad. Naz. Lincei 14, 5-11, 2003.

6. L. Báez-Duarte, A Class of Invariant Unitary Operators. Adv. Math. 144 (1999) 1-12.

7. M. Balazard, E. Saias, Notes sur la fonction $\zeta$ de Riemann 4, Adv. Math. 188 (2004) 69-86.

8. C. Constara, T. Ransford, Which de Branges-Rovnyak spaces are Dirichlet spaces (and vice versa)?. J. Funct. Anal. 265(12), 3204-3218 (2010)

9. E. Fricain, J. Mashreghi, The theory of $\mathcal{H}_{b}$ spaces. Vol. 1, volume 20 of New Mathematical Monographs. Cambridge University Press, Cambridge (2016)

10. E. Fricain, J. Mashreghi, The theory of $\mathcal{H}_{b}$ spaces. Vol. 2, volume 21 of New Mathematical Monographs. Cambridge University Press, Cambridge (2016)

11. H. Hedenmalm, P. Lindqvist, and K. Seip. A Hilbert space of Dirichlet series and systems of dilated functions in $L^{2}(0,1)$. Duke Math. J., 86:137, 1997. MR 99i:42033

12. H. Hedenmalm, B. Korenblum, K. Zhu, Theory of Bergman spaces. GTM Springer, volume 199, 2000.

13. J. Mashreghi, K. Kellay, Omar El-Fallah, and T. Ransford, A primer on the Dirichlet space. Cambridge Tracts in Mathematics (203), Cambridge University Press, 2014.

14. H. L. Montgomery, R. C. Vaughan, Multiplicative number theory: 1. Classical theory. Cambridge Studies in Advanced Mathematics (97), Cambridge University Press, 2006.

15. N. Nikolski, In a shadow of the RH: cyclic vectors of the Hardy spaces on the Hilbert multidisc. Ann. Inst. Fourier, 62(5), 1601-1626 (2012).

16. B. Nyman, On some groups and semigroups of translations. Thesis, Uppsala, 1950.

17. D. Sarason, Unbounded Toeplitz operators. Integr. Equ. Oper. Theory. 61(2), 281-298 (2008).

18. D. Sarason, Sub-Hardy Hilbert Spaces in the Unit Disk, John Wiley \& Sons Inc., New York, 1994.

19. A. Wintner, Diophantine approximation and Hilbert's space. Amer. J. Math. 66 (1944), p.564578.

imeCC, Universidade Estadual de Campinas, Campinas-SP, Brazil.

E-mail address: waleed@ime.unicamp.br 